\title{
An unusual intracardiac shunt secondary to penetrating cardiac trauma
}

\author{
W J C Hobbs, B Clarke, N J Odom
}

Manchester Heart

Centre, Royal

Infirmary, Oxford

Road, Manchester M13

9WL, UK

W J C Hobbs

B Clarke

N J Odom

Correspondence to: Dr Clarke.

Accepted for publication 12 December 1997
A 22 year old man was admitted to the accident and emergency department having sustained a $5 \times 3 \mathrm{~cm}$ knife wound to the right anterior chest wall in the fourth intercostal space. He was haemodynamically stable on admission but dyspnoeic at rest. Pulse was 110 beats/min and blood pressure $106 / 60 \mathrm{~mm} \mathrm{Hg}$. The only abnormality on examination was dullness to percussion on the right chest wall; heart sounds were normal. A plain chest $x$ ray confirmed a right haemothorax. Three litres of blood were drained using an intercostal drain. A median sternotomy was performed; the right internal thoracic artery, which had been transected, was ligated. A small laceration in the right atrioventricular groove was sutured.

The patient made an unremarkable recovery and was discharged home. He was reviewed three weeks later when examination revealed a pansystolic murmur over the whole precordium. A transthoracic echocardiogram showed a high velocity jet from the left ventricle to the right atrium. Transoesophageal echocardiography at a depth of $30 \mathrm{~cm}$ and in multiple planes demonstrated a laceration in the junction of the aortomitral continuity and the intra-atrial septum (figs 1 and 2). Colour flow mapping confirmed high velocity flow from the left ventricular outflow tract to right and left atria.

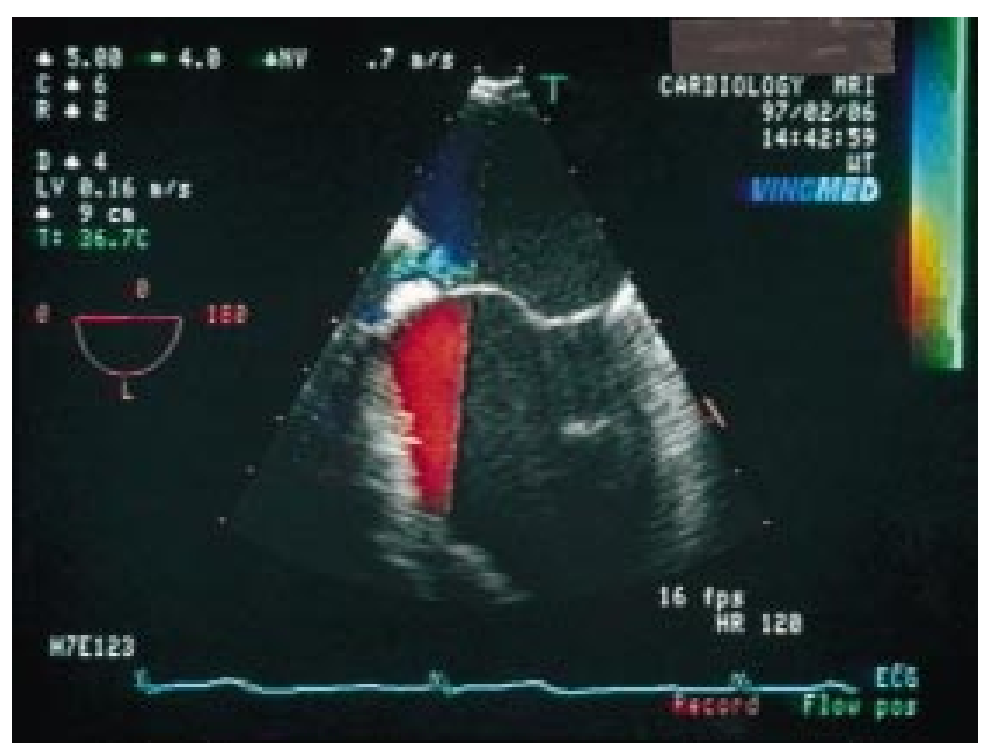

He has remained well over the past 12 months and has been managed conservatively with regular follow up and advice about prophylactic antibiotics.

\section{Discussion}

Penetrating cardiac wounds are increasingly common, and advances in initial resuscitation and surgical management mean more patients are surviving the initial insult. There are often several lesions, and initial assessment in hypotensive patients, particularly with surgical emphysema, may not reveal all injuries. ${ }^{1}$ Others suggest that small defects may grow following fibrous retraction of wound edges, resolution of oedema, ventricular enlargement, and lysis of occluding clot, therefore becoming apparent at a later date. ${ }^{2}$ Stabilisation and surgical correction of life threatening lesions takes priority and this often means that the heart is not fully explored surgically. Earlier series have demonstrated numerous late sequelae including atrial and ventricular septal defects, tricuspid and mitral valve lacerations, and coronary artery injury. The reported incidence of late sequelae of $20 \%$ suggests that comprehensive postoperative evaluation is essential for all patients. ${ }^{3}$ Not all of these defects are haemodynamically significant and may require only observation and advice regarding prophylactic antibiotics.

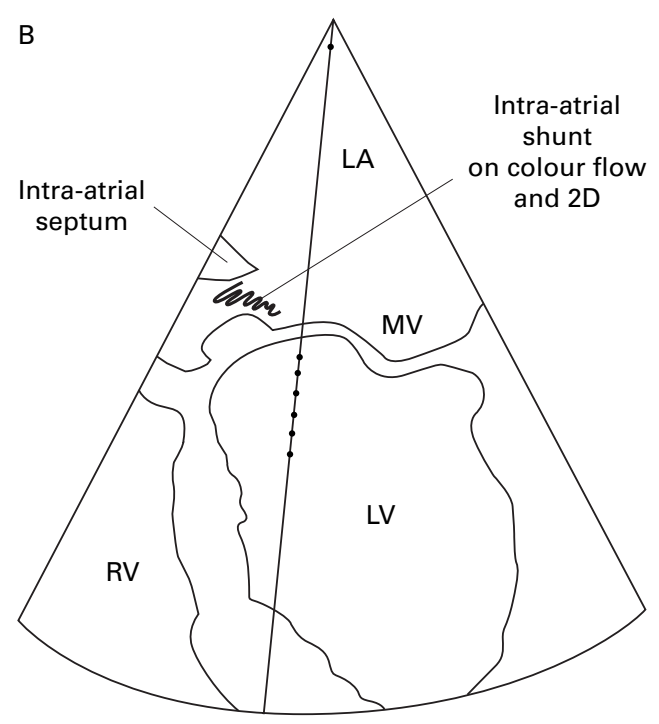

Figure 1 Left atrial (LA) to right atrial shunt. MV. Mitral valve; RV, right ventricle; LV, left ventricle. 


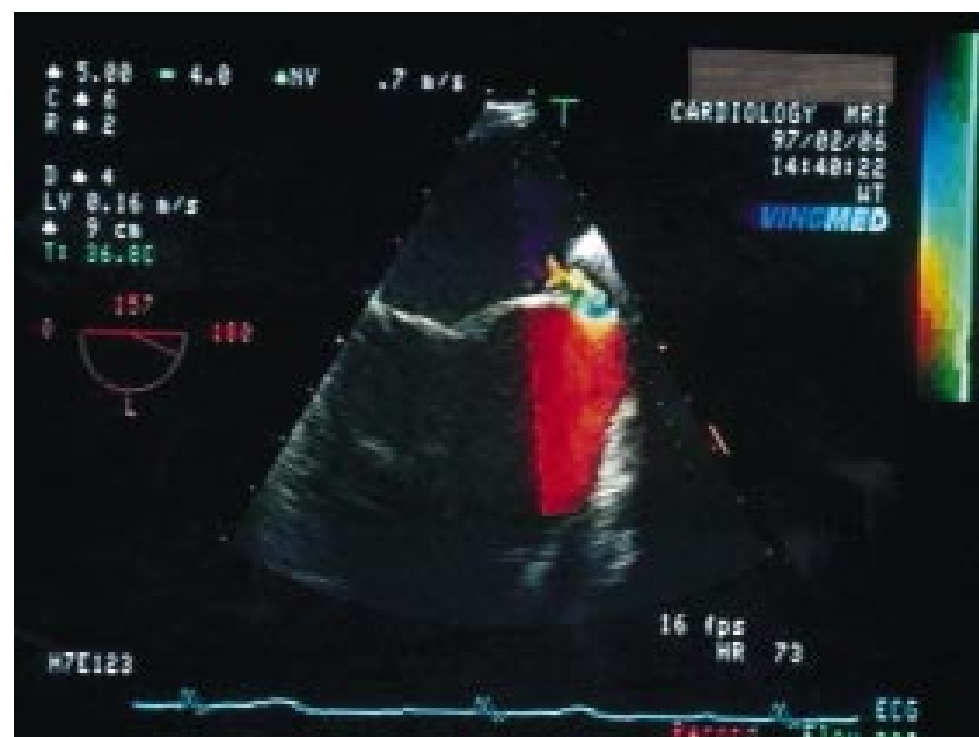

Figure 2 Left ventricular outflow tract (LVOT) to left atrium (LA) shunt. LV, left ventricle.

Those that do require further surgery can be closed electively with little mortality or morbidity. ${ }^{1}$

Knife and bullet wounds at various levels of the interventricular and atrioventricular septum producing left ventricular to right ventricular or left ventricular to right atrial shunts are well described. ${ }^{4}$ To our knowledge survival with a left ventricular to left and right atrial shunt caused by a single knife wound is unique. Our case shows the clinical usefulness of transoesophageal echocardiography in detecting and fully delineating these lesions. It also demonstrates the anatomy of this area well, and

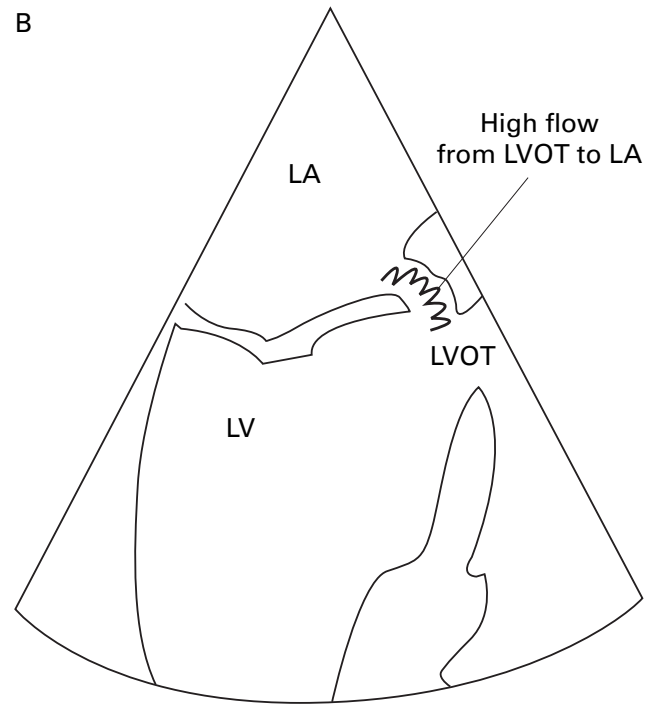

reinforces the importance of echocardiography in the follow up of patients. To define fully the anatomical abnormality in this patient, omniplane transoesophageal echocardiography was essential.

1 Demtriades D, Van der Veen BW. Penetrating injuries of the heart: experience over 2 years in South Africa. 7 Trauma 1983;23:1034-41.

2 Demetriades D. Cardiac penetrating injuries: personal experience of 45 cases. Br f Surg 1984;71:95-7.

3 Cha EK, Mittal V, Allaben RD. Delayed sequelae of penetrating cardiac injury. Arch Surg 1993;128:836-41.

4 Asfaw I, Thomas NW, Arbulu A. Intraventricular septal defects from penetrating injuries of the heart. $\mathcal{F}$ Thorac Cardiovasc Surg 1975;69:450-7. 